\title{
UAV Behavior-Intention Estimation Method Based on 4-D Flight-Trajectory Prediction
}

\author{
Honghai Zhang ${ }^{1, *}$, Yongjie Yan ${ }^{2}$, Shan $\mathrm{Li}^{1}{ }^{1}$, Yuxin $\mathrm{Hu}^{2}$ and Hao Liu ${ }^{1}$ \\ 1 College of Civil Aviation, Nanjing University of Aeronautics and Astronautics, Nanjing 210016, China; \\ lishan0812@nuaa.edu.cn (S.L.); hliu@nuaa.edu.cn (H.L.) \\ 2 State Key Laboratory of Air Traffic Management System and Technology, Nanjing 210007, China; \\ yanyongjie41403@sina.com (Y.Y.); sklatm@cetc.com.cn (Y.H.) \\ * Correspondence: honghaizhang@nuaa.edu.cn
}

\section{check for} updates

Citation: Zhang, H.; Yan, Y.; Li, S.; $\mathrm{Hu}$, Y.; Liu, H. UAV

Behavior-Intention Estimation

Method Based on 4-D

Flight-Trajectory Prediction. Sustainability 2021, 13, 12528. https://doi.org/10.3390/ su132212528

Academic Editors: Xiaobei Jiang, Haixiang Lin, Fei Yan and Qian Cheng

Received: 17 September 2021 Accepted: 8 November 2021 Published: 12 November 2021

Publisher's Note: MDPI stays neutral with regard to jurisdictional claims in published maps and institutional affiliations.

Copyright: (c) 2021 by the authors. Licensee MDPI, Basel, Switzerland. This article is an open access article distributed under the terms and conditions of the Creative Commons Attribution (CC BY) license (https:// creativecommons.org/licenses/by/ $4.0 /)$.

\begin{abstract}
Aiming at the limitation of the traditional four-dimensional (4-D) trajectory-prediction model of unmanned aerial vehicles (UAV), a 4-D trajectory combined prediction model based on a genetic algorithm is proposed. Based on historical flight data and the UAV motion equation, the model is weighted dynamically by a genetic algorithm, which can predict UAV trajectory and the time of entering the protection zone instantly and accurately. Then, according to the number of areas where the tangent line of the current trajectory point intersects with the collision area, alarm area, alert area, and the time of entering the protection zone, the UAV's behavior intention can be estimated. The simulation experiments verify the dangerous behaviors of UAV under different danger levels, which provides reference for the subsequent maneuvering strategies.
\end{abstract}

Keywords: UAV; 4-D flight trajectory; SVR; combined prediction; behavior intention

\section{Introduction}

With the rapid development of UAV technology, UAV have shown more and more irreplaceable advantages, playing a pivotal role in equipment monitoring, agriculture, forestry and plant protection, public safety, search and rescue, disaster assistance, meteorological monitoring, and cargo transportation [1]. However, the large-scale operation of UAV in the future will inevitably be accompanied by operational risks that cannot be ignored, which will bring new challenges to current air-traffic management. Therefore, in view of the requirements of $U A V$ control technology, it is urgent to study the problem of UAV behavior-intention estimation.

UAV behavior-intention estimation is the process of identifying the behavior of UAV through the technology of flight-trajectory prediction to monitor operational safety risks. In the aspect of trajectory prediction, Yang Rennong et al. [2] proposed a trajectory-prediction model based on the Bi-LSTM neural network, which has higher prediction accuracy compared with the model based on the Elman neural network. Benavides et al. [3] proposed a trajectory-prediction algorithm that can reduce flight fuel consumption by comprehensively considering aircraft level and height profiles, planned flight time, and integrated fuel consumption. Wu Qinggang [4] proposed the RVFLNN short-time flight trajectory-prediction algorithm for cooperative $U A V$, which realized that fast and real-time prediction of UAV position informs action. Liang Shaojun et al. [5] proposed a multi-working-condition prediction model with UAV pitch angle, elevator deflection angle, cylinder temperature, and engine speed as the prediction variables. Compared with the single-working-condition prediction model, it is more suitable for the multi-working-condition characteristics of UAV. Considering the uncertain factors in flight, Li Teng et al. [6] used the geometric method to predict the 4-D flight trajectory of UAV. Wang Yiping et al. [7] established state space equation and estimated UAV attitude by particle-filtering algorithm to realize UAV trajectory prediction. Tastamberkov, Hong et al. [8,9] regarded flight-trajectory prediction 
as a regression problem and realized the prediction of flight trajectory by using the wavelet decomposition and multiple logistic regression model, respectively. A single model with different emphases may overgeneralize the prediction of the flight trajectory. However, time dimension is not considered in trajectory prediction, which has some limitations.

In this paper, a 4-D combined flight-trajectory prediction model is proposed. According to the historical flight data and motion equation of UAV, the combined prediction of longitude, latitude, altitude and the time of entering the protection zone was made by genetic algorithm. Based on the results of flight-trajectory prediction, state parameters, such as the time of entering the protection zone and crossing-point number, were extracted, and the classification criterion of dangerous behavior was established to estimate the behavior intention. The simulation results showed that the model can accurately predict the 4-D flight trajectory and identify the dangerous behavior level, which is of great significance to ensure operation safety.

\section{4-D Trajectory-Prediction Model of UAV}

\subsection{Trajectory-Prediction Model Based on Flight Data}

\subsubsection{SVR Algorithm Principle}

Support vector machine regression (SVR) is a regression algorithm derived from the support vector machine (SVM) classification algorithm, which is suitable for small samples. The basic idea is to find an optimal hyperplane so that the distance between all sample points and the hyperplane is minimal. That is, the total deviation between all sample points and the hyperplane is minimal [10-12]. Suppose there is a set of sample data, $\left(x_{1}, y_{1}\right), \cdots,\left(x_{n}, y_{n}\right)$. When the regression problem is nonlinear, a kernel function should be introduced to map the nonlinear sample data into a high-dimensional space to linearize it so that linear regression can be carried out. In this paper, the Gaussian radial basis kernel function is selected.

$$
K\left(x, x_{i}\right)=\exp \left(-\frac{\left\|x-x_{i}\right\|^{2}}{2 \sigma^{2}}\right)=\exp \left(-\mathrm{g}\left\|x-x_{i}\right\|^{2}\right),
$$

where $g$ is the kernel function parameter. After mapping through the kernel function, the sample data are linear in the high-dimensional space, so there must be a linear regression function.

$$
f\left(x_{i}\right)=<w, K\left(x, x_{i}\right)>+b,
$$

where $w$ is the weight vector and $b$ is the offset quantity. Based on the principle of structural risk minimization, $w$ and $b$ are obtained by solving the following optimization problems.

$$
\begin{aligned}
& \min _{w, b, \tilde{\xi}_{i}, \widetilde{\xi}_{i}} \frac{1}{2}\|w\|^{2}+C \sum_{i=1}^{m}\left(\xi_{i}+\widetilde{\xi}_{i}\right), \\
& \text { s.t. }\left\{\begin{array}{l}
f\left(x_{i}\right)-y_{i} \leq \varepsilon+\xi_{i} \\
y_{i}-f\left(x_{i}\right) \leq \varepsilon+\widetilde{\xi}_{i} \\
\xi_{i}, \widetilde{\xi}_{i} \geq 0
\end{array},\right.
\end{aligned}
$$

where $C$ is the penalty factor and $C>0$, and $\xi_{i}, \widetilde{\zeta}_{i}$ is slack factor to avoid the trained fitting, and $\varepsilon$ is the upper limit of allowable error.

\subsubsection{SVR 4-D Trajectory-Prediction Algorithm}

The flight trajectory of the UAV is the location information with a time stamp, including longitude, latitude, and altitude, namely $\left(t_{i}, x_{t_{i}}, y_{t_{i}}, z_{t_{i}}\right), i=1,2, \cdots$, where $t_{i}$ is the record time of the trajectory point and $x_{t_{i}}, y_{t_{i}}$ and $z_{t_{i}}$ are the longitude, latitude, and altitude information of the location, respectively, at time, $t_{i}$. A time window $\left[t_{i-n}, t_{i-1}\right]$ of length $n$ was designated, and the information of longitude, latitude, and altitude in the time window was regarded as three mutually independent input variables. The longitude, 
latitude, and altitude at $t_{i}$ were taken as output variables to train the SVR model. The steps of the SVR algorithm are as follows:

Step 1: Data processing. The historical flight-trajectory data set under a certain mission scene is acquired in real time from ADS-B of UAV. However, due to signal loss, statistical error, and other reasons, there are often abnormal and missing data in the original data set. Therefore, Lagrange interpolation, wavelet threshold denoising, and other methods are needed to preprocess the original trajectory data. In order to accelerate the model optimization speed and improve the prediction accuracy, the following formula is used to normalize the preprocessed data and make it normalized to the interval $[-1,1]$.

$$
x^{\prime}=\frac{2\left(x-x_{\min }\right)}{x_{\max }-x_{\min }}-1,
$$

where $x$ is the preprocessed trajectory data and, $x^{\prime}$ is the normalized data, and $x^{\prime} \in[-1,1]$ and $x_{\max }, x_{\min }$ are the maximum and minimum values in the selected data, respectively.

Step 2: Algorithm input. The future flight trajectory is the result of the interaction between the present and the historical trajectory. The further the historical trajectory point is from the current time, $t_{i}$, the less correlated it is. In this paper, a memoryless prediction method is used. By delimiting a time window, $\left[t_{i-n}, t_{i-1}\right]$, of length $n$, the trajectory at $t_{i}$ is comprehensively predicted through the trajectory points at $t_{i-n}, t_{i-n+1}, \cdots, t_{i-1}$. The data of longitude, latitude, and altitude in this time window are regarded as three mutually independent variables as the input of the algorithm. For example, the input vector of the longitude component is $\left(x_{t_{i-n}}^{\prime}, x_{t_{i-n+1}}^{\prime}, \cdots, x_{t_{i-1}}^{\prime}\right)$.

Step 3: Parameter selection. When the input data are used to train the SVR model, two important parameters should be properly selected: penalty factor, $C$, and kernel function parameter, $g$. In order to find the optimal parameter combination $(C, g)$, the grid-search method is adopted in this paper. The minimum mean square error (MSE) is taken as the goal to search and obtain the optimal parameter combination $(C, g)$.

Step 4: Parallel prediction. Through Step 3, the optimal parameter combinations corresponding to longitude, latitude, and altitude, $\left(C_{x}, g_{x}\right),\left(C_{y}, g_{y}\right)$, and $\left(C_{z}, g_{z}\right)$, were obtained, then substituted into the training model of the SVR algorithm. The obtained three models were used to predict the trajectory information of the UAV simultaneously. With the UAV flying, the time window constantly changes, and the optimal parameter combination and prediction model are updated in real time.

\subsection{Trajectory-Prediction Model Based on Motion Equation}

Based on ADS-B data, the position, speed, heading angle, and other parameters of the aircraft at every moment can be obtained. Since the speed of the aircraft can be obtained each time, the motion state between two adjacent measurements can be regarded as the linear motion with uniform variable speed. The data of UAV obtained at $t_{i}$ moment by transformation are $X\left(t_{i}\right), Y\left(t_{i}\right), Z\left(t_{i}\right), V_{l}\left(t_{i}\right), V_{v}\left(t_{i}\right), \beta\left(t_{i}\right), \theta\left(t_{i}\right)$, and $T\left(t_{i}\right)$, where $X\left(t_{i}\right), Y\left(t_{i}\right)$ take the ADS-B receiving end as coordinates center, the geomagnetic north pole and its vertical eastward direction are the values of the $\mathrm{X}$ and $\mathrm{Y}$ axes, respectively, $\mathrm{Z}\left(t_{i}\right)$ is the height, $V_{l}\left(t_{i}\right)$ is the horizontal velocity, $V_{v}\left(t_{i}\right)$ is the vertical velocity, $\beta\left(t_{i}\right)$ is the horizontal heading angle, $\theta\left(t_{i}\right)$ is the vertical heading angle, and $T$ is the sampling-time interval. Each $t_{i}$ corresponds to a unique and determined UAV position, heading angle, and speed [13]. The motion state of UAV can be expressed as: $W\left(t_{i}\right)=\left[X\left(t_{i}\right), Y\left(t_{i}\right), Z\left(t_{i}\right), V_{x}\left(t_{i}\right), V_{y}\left(t_{i}\right), V_{z}\left(t_{i}\right), a_{x}, a_{y}, a_{z}\right]^{T}$, and the UAV kinematic coordinate system is shown in Figure 1 . The motion equation of the UAV can be established as the following formula.

$$
\left\{\begin{array}{l}
W\left(t_{i+1}\right)=W\left(t_{i}\right)+V\left(t_{i}\right) \times T+\frac{1}{2} a T^{2} \\
V\left(t_{i+1}\right)=V\left(t_{i}\right)+a T
\end{array} .\right.
$$


In Equation (6), it can be known that $a=\frac{V\left(t_{i+1}\right)-V\left(t_{i}\right)}{T}$. Equation (6) can be rewritten as the following formula.

$$
W\left(t_{i+1}\right)=W\left(t_{i}\right)+\frac{V\left(t_{i}\right)+V\left(t_{i+1}\right)}{2} T .
$$

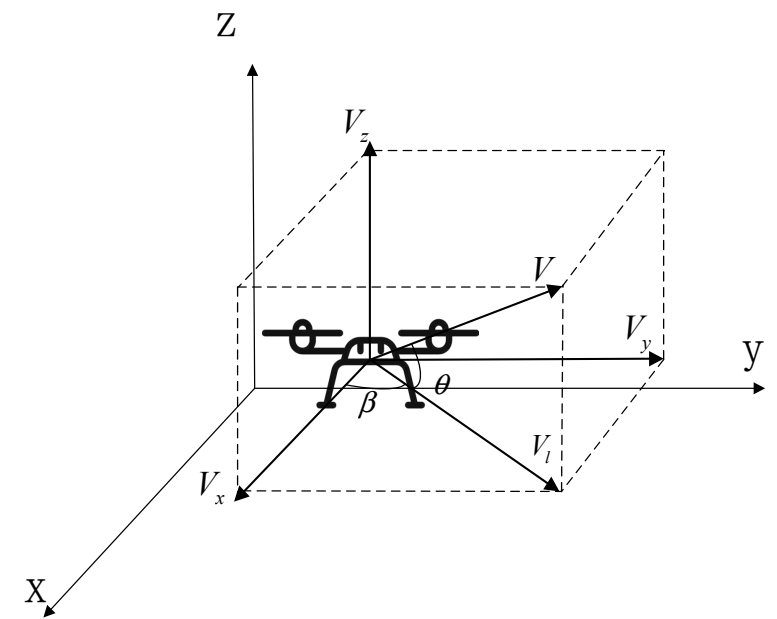

Figure 1. UAV kinematic coordinate system.

Based on the above information, the trajectory equations of the UAV on X, Y, and Z axes can be established:

$$
\left\{\begin{array}{l}
X\left(t_{i+1}\right)=X\left(t_{i}\right)+V_{x}\left(t_{i}\right) \times T+\frac{1}{2} a_{x}\left(t_{i}\right) T^{2} \\
Y\left(t_{i+1}\right)=Y\left(t_{i}\right)+V_{y}\left(t_{i}\right) \times T+\frac{1}{2} a_{y}\left(t_{i}\right) T^{2} \\
Z\left(t_{i+1}\right)=Z\left(t_{i}\right)+V_{z}\left(t_{i}\right) \times T+\frac{1}{2} a_{z}\left(t_{i}\right) T^{2} \\
V_{x}\left(t_{i+1}\right)=V_{x}\left(t_{i}\right)+a_{x}\left(t_{i}\right) \times T \\
V_{y}\left(t_{i+1}\right)=V_{y}\left(t_{i}\right)+a_{y}\left(t_{i}\right) \times T \\
V_{z}\left(t_{i+1}\right)=V_{z}\left(t_{i}\right)+a_{z}\left(t_{i}\right) \times T \\
V_{x}\left(t_{i}\right)=V_{l}\left(t_{i}\right) \times \cos \left(\beta\left(t_{i}\right)\right) \\
V_{y}\left(t_{i}\right)=V_{l}\left(t_{i}\right) \times \sin \left(\beta\left(t_{i}\right)\right) \\
V_{z}\left(t_{i}\right)=V_{v}\left(t_{i}\right) \times \tan \left(\beta\left(t_{i}\right)\right)
\end{array} .\right.
$$

Equation (8) can be rewritten as follows:

$$
\left\{\begin{array}{l}
X\left(t_{i+1}\right)=X\left(t_{i}\right)+\frac{1}{2}\left(V\left(t_{i}\right) \times \cos \left(\beta\left(t_{i}\right)\right)+V_{l}\left(t_{i-1}\right)\right) \times \cos \left(\beta\left(t_{i-1}\right)\right) \times T \\
Y\left(t_{i+1}\right)=X\left(t_{i}\right)+\frac{1}{2}\left(V\left(t_{i}\right) \times \sin \left(\beta\left(t_{i}\right)\right)+V_{l}\left(t_{i-1}\right)\right) \times \sin \left(\beta\left(t_{i-1}\right)\right) \times T \\
Z\left(t_{i+1}\right)=X\left(t_{i}\right)+\frac{1}{2}\left(V\left(t_{i}\right) \times \tan \left(\theta\left(t_{i}\right)\right)+V_{v}\left(t_{i-1}\right)\right) \times \tan \left(\theta\left(t_{i-1}\right)\right) \times T
\end{array} .\right.
$$

In Equation (9), $X\left(t_{i}\right), Y\left(t_{i}\right), Z\left(t_{i}\right), \beta\left(t_{i}\right)$, and $\theta\left(t_{i}\right)$ are the UAV data obtained from ADS-B. $X\left(t_{i+1}\right)$ is the predicted longitude, $Y\left(t_{i+1}\right)$ is the predicted latitude, and $Z\left(t_{i+1}\right)$ is the predicted altitude.

\subsection{Combined Prediction Model Based on Genetic Algorithm}

4-D flight-trajectory prediction and motion-equation flight-trajectory prediction models are mentioned in Sections 2.1 and 2.2. In this section, the two prediction methods are combined, and the combination principle is applied to improve the prediction of UAV flight trajectory so as to improve the prediction accuracy. The genetic algorithm is based on Darwin's evolution theory and simulates the process of natural selection and survival of the fittest. Through selection, replication, mutation, crossover, and other operations, the population evolves in the optimal direction continuously, and the approximate optimal solution of the problem can be finally obtained [14-16]. 
In the combined prediction, the genetic algorithm unifies the two prediction models into one combined model by finding the optimal weight parameter $\left[\omega_{1}, \omega_{2}\right]$ of the two prediction models.

$$
M(T)=\omega_{1} m_{1}(T)+\omega_{2} m_{2}(T),
$$

where $m_{a}(t), \omega_{a}$ are the predicted results of the 4-D trajectory prediction model and the assigned weight, respectively, $m_{b}(t), \omega_{b}$ are the predicted results of the motion-equation prediction model and the assigned weight, respectively, $\omega_{1}+\omega_{2}=1$, and $M(T)$ is the weighted combined prediction results.

(1) Individual fitness function

Since the relative error between the predicted value and the actual value is required to be as small as possible, the objective function of the genetic algorithm is the relative error.

$$
W_{n}=\frac{|G(T)-M(T)|}{G(T)},
$$

where $G(T)$ is the actual value of trajectory at the time, $\mathrm{T}$, and $W_{n}$ is the relative error of an individual $n$.

In the genetic algorithm, it is generally believed that the higher the fitness value is, the greater the possibility of the survival of the individual is. That is, the genes within the individual are relatively more excellent. Therefore, the fitness value of the corresponding individual is the reciprocal of the objective function.

$$
f(n)=\frac{1}{W_{n}} .
$$

(2) Individual coding

The individual is the value of two weights $\left[\omega_{1}, \omega_{2}\right]$, and the $\omega$ is in the interval of $(0,1)$. Therefore, it is encoded by two integers; the value is between $\{100,999\}$. Therefore, there is a mapping relationship between gene $\omega_{i}^{\prime}$ and weight $\omega_{i}$ as follows:

$$
\omega_{i}=\frac{\omega_{i}^{\prime}}{\sum_{i=1}^{2} \omega_{i}^{\prime}}
$$

\section{(3) Selection}

In this paper, the next generation was selected by way of tournament, and the individuals ranked in the bottom 20 in fitness were randomly eliminated, while the individuals ranked in the top 20 in fitness were randomly copied. The number of the eliminated individuals was the same as the number of the copied individuals so as to ensure that the excellent genes were retained and the total number of the population remained unchanged.

(4) Crossover

By means of sampling with replacement, any two individuals are selected from the population for crossover. The crossover adopts fragment intersecting. First, a random number $[0,1]$ is generated. If the number is less than the crossover probability, the random starting point and ending point of the crossover are generated. The DNA fragments in the same position are intercepted from two individuals, and the DNA fragments are exchanged, respectively.

(5) Mutation

Each gene in an individual is formed into a random number [0,1]. If the number is less than the mutation probability, a number $[0,9]$ is randomly selected to replace the gene. 


\subsection{Prediction of Time Entering the Protection Zone}

The protection zone, a convex polyhedron with the same cross-section, is a special airspace that UAV are forbidden to enter. Predicting the time of entering protection zone is an important part of evaluating the intention of UAV's behavior. This article take the trajectory reckoning algorithm to calculate the time of entering protection zone. At the current moment $t_{i}$, trajectory points $\left(t_{i+1}, x_{t_{i+1}}, y_{t_{i+1}}, z_{t_{i+1}}\right), \cdots,\left(t_{i+m}, x_{t_{i+m}}, y_{t_{i+m}}, z_{t_{i+m}}\right)$ at $m$ subsequent moments are predicted. Find the time corresponding to the first point entering the protection zone in the $m$ trajectory points, so as to calculate the time of entering the protection zone.

Suppose the height range of a protection zone is $\left[h_{1}, h_{2}\right]$, the cross-section is a convex pentagon. $P_{1}, P_{2}, \cdots, P_{5}$ are the vertices of the convex hull respectively, and $L_{1}, L_{2}, \cdots, L_{5}$ are the lines where the edges of the convex hull are respectively. The conditions for judging the UAV entering the protection zone are as follows: the predicted trajectory point of the UAV enters the cross-section convex hull of the protection zone, and the height of the point is within the height range of the protection zone. Figure 2 shows the front view and top view of the protection zone and UAV trajectory points.

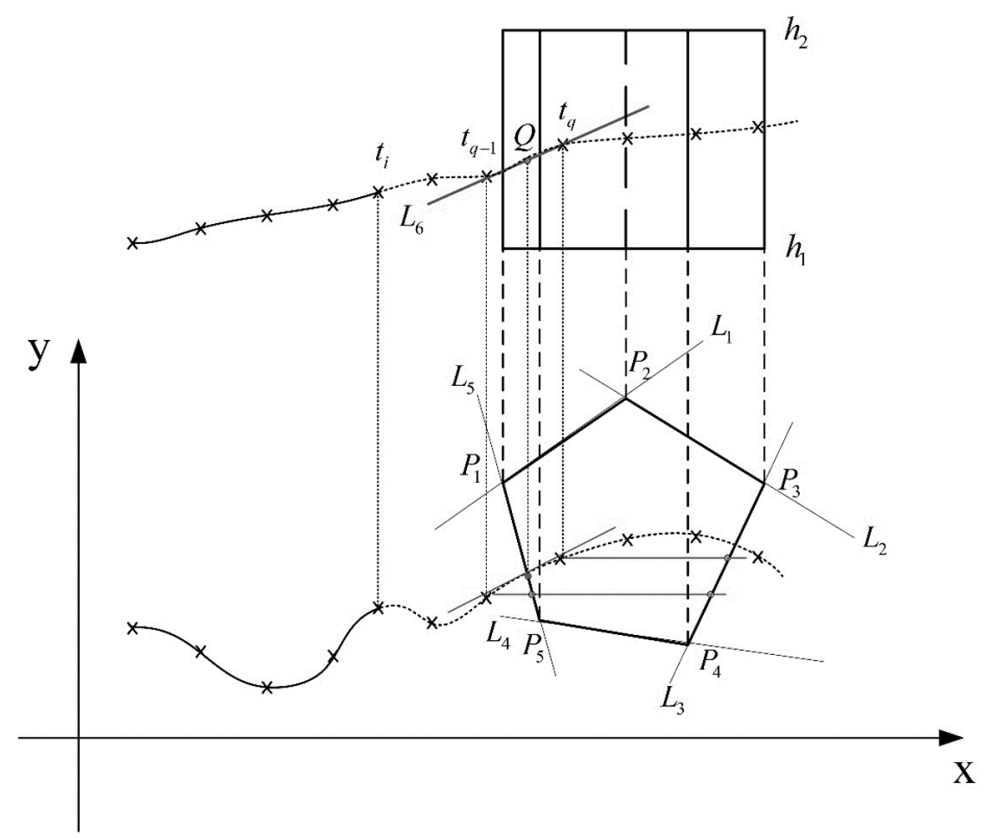

Figure 2. Prediction of time entering the protection zone.

First, judge whether the predicted trajectory point $\left(t_{q}, x_{t_{q}}, y_{t_{q}}, z_{t_{q}}\right)$ is located in the cross-section. Take the trajectory point as the vertex and draw a horizontal ray to the right of it, as the rays of the trajectory point at time $t_{q}$ and $t_{q-1}$ in the figure above. Set the ray equation at time $t_{q}^{\prime}$ as

$$
y=y_{t_{q}}, x \in\left[x_{t_{q}}, \infty\right] .
$$

The equation of the line where the edge of the cross-section is

$$
y=k_{i} x+b_{i} \quad(i=1,2, \cdots, 5) .
$$

Then, the intersection point of ray and cross-section is

$$
\left\{\begin{array}{l}
x_{j}=\frac{y_{t_{q}}-b_{i}}{k_{i}} \\
y_{j}=y_{t_{q}}
\end{array}, j \in[0,5] .\right.
$$


Since the intersection point of the ray and the convex hull may be on the extension line of the edge, the following equation is used to judge whether the intersection point is on the cross-section.

$$
\begin{gathered}
\left\{\begin{array}{l}
\min x_{P_{n}} \leq x_{j} \leq \max x_{P_{n}} \\
\min y_{P_{n}} \leq y_{j} \leq \max y_{P_{n}}
\end{array}, j \in[0,5], n=1,2, \cdots 5,\right. \\
r_{j}=\left\{\begin{array}{l}
0,\left(x_{j}, y_{j}\right) \text { on the edge of the convex hull } \\
1,\left(x_{j}, y_{j}\right) \text { not on the edge of the convex hull }
\end{array} .\right.
\end{gathered}
$$

The number of rays intersecting the cross-section, $R_{j}$, is

$$
R_{j}=\sum_{j=1}^{5} r_{j}
$$

If $R_{j}$ is odd, the point is in the cross-section. If $R_{j}$ is even, it is outside the cross-section. For the trajectory points within the cross-section, judge whether they are within the height range of the protection zone

$$
h_{1} \leq z_{t_{i}} \leq h_{2} .
$$

If the point is the first trajectory point of entering the protection zone, the corresponding time is $t_{q}$, and the previous time is $t_{q-1}$. In the cross-section, the line, $L_{6}$, of the trajectory point at the time of $t_{q}$ and $t_{q-1}$ is

$$
\frac{x-x_{q-1}}{x_{q}-x_{q-1}}=\frac{y-y_{q-1}}{y_{q}-y_{q-1}} .
$$

The intersecting edge of the line and the convex hull is $y=k_{i} x+b_{i}$. The intersection point $Q\left(x_{1}, y_{1}\right)$ can be obtained by combining it with the above equation. The space linear equation of the trajectory point is

$$
\frac{x-x_{q-1}}{x_{q}-x_{q-1}}=\frac{y-y_{q-1}}{y_{q}-y_{q-1}}=\frac{z-z_{q-1}}{z_{q}-z_{q-1}} .
$$

Substitute the intersection point $Q\left(x_{1}, y_{1}\right)$ into the above equation to get the height of the intersection point, $z_{1}$. Set the Euclidean distance between $Q$ and trajectory point at the time, $t_{q-1}$, as $d_{1}$

$$
d_{1}=\sqrt{\left(x_{1}-x_{q-1}\right)^{2}+\left(y_{1}-y_{q-1}\right)^{2}+\left(z_{1}-z_{q-1}\right)^{2}} .
$$

The velocity, $v$, of the UAV between the two predicted trajectory points is regarded as a constant

$$
v=\frac{\sqrt{\left(x_{q}-x_{q-1}\right)^{2}+\left(y_{q}-y_{q-1}\right)^{2}+\left(z_{q}-z_{q-1}\right)^{2}}}{t_{q}-t_{q-1}} .
$$

Then, the estimated time, $T_{1}$, of entering the protection zone at $t_{i}$ is

$$
T_{1}=t_{q-1}+\frac{d_{1}}{v} .
$$

\section{A Method for Estimating UAV Behavior Intention}

Based on the 4-D flight-trajectory prediction of the UAV and according to the state parameters, such as the number of intersection points between the current trajectory point tangent line and the protection zone, and the time of entering the protection zone, the behavior-intention estimation model is proposed. The implementation steps of the model are as follows. 
Step 1: Calculate the intersection point of the tangent projection of the current trajectory point on the horizontal plane and the line where each edge of the cross-section of the protection zone is. Set the tangent projection equation as

$$
y=k x+b .
$$

Tangent projection can happen in one of two ways.

(1) When $k=k_{i}$ and $b=b_{i}$, the tangent projection coincides with a certain side of the cross-section.

(2) When $k \neq k_{i}$ or $b \neq b_{i}$, according to Equation (15), solve $n$ binary equations of linear equations of first order, which are composed of the tangent projection and the line where each edge of the cross-section is located, and obtain the coordinates $\left(X_{m}, Y_{m}\right)$ of the intersection point of the tangent projection and the line where each edge of the cross-section is located.

$$
\left\{\begin{array}{l}
X_{m}=\frac{b_{i}-b}{k-k_{i}} \\
Y_{m}=\frac{k b_{i}-k_{i} b}{k-k_{i}}
\end{array} \quad(m=1,2, \cdots, n) .\right.
$$

Step 2: Calculate the number of intersection points between tangent line and protection zone planes. The equation of the tangent line is

$$
\frac{x-x_{t_{i}}}{a}=\frac{y-y_{t_{i}}}{b}=\frac{z-z_{t_{i}}}{c} .
$$

According to Step 1 , if the condition of case 1 is satisfied, the endpoint coordinates, $\left(X_{1}, Y_{1}\right),\left(X_{2}, Y_{2}\right)$, of the overlapping edges of the cross-section are substituted into the equation of the tangent line to obtain the height, $Z_{1}, Z_{2}$, of the endpoints. If $Z_{1}, Z_{2} \in\left[h_{1}, h_{2}\right]$, there are numerous intersection points between the tangent line and the protection zone. If $Z_{1}, Z_{2}$ has only one height equal to the upper or lower limit of the protection zone, then there is only one intersection point. If $Z_{1}, Z_{2} \notin\left[h_{1}, h_{2}\right]$, there is no intersection point.

If the condition of case 2 is satisfied, the intersection point $\left(X_{m}, Y_{m}\right)$ is substituted into the above equation to obtain the height of the intersection point, $Z_{m}$. It is assumed that there are $N$ intersection points, and the function $P_{n}$ is used to judge whether each intersection point is on the protection zone planes.

$$
P_{n}=\left\{\begin{array}{l}
1,\left(X_{m}-X_{p}\right)\left(X_{m}-X_{q}\right) \leq 0 \text { and } Z_{m} \in\left[h_{1}, h_{2}\right] \\
0,\left(X_{m}-X_{p}\right)\left(X_{m}-X_{q}\right)>0 \text { or } Z_{m} \notin\left[h_{1}, h_{2}\right]
\end{array},\right.
$$

where $X_{p}, X_{q}$ are the abscissas of two adjacent vertices in the cross-section. When $P_{n}=1$, the intersection point is on the protection zone. The number of intersection points between the tangent line and the protection zone is $Q=\sum_{n=1}^{N} P_{n}$. If $Q>0$, the tangent line intersects the protection zone.

The flow chart of UAV behavior-intention estimation method based on 4-D flighttrajectory prediction is shown in Figure 3. 


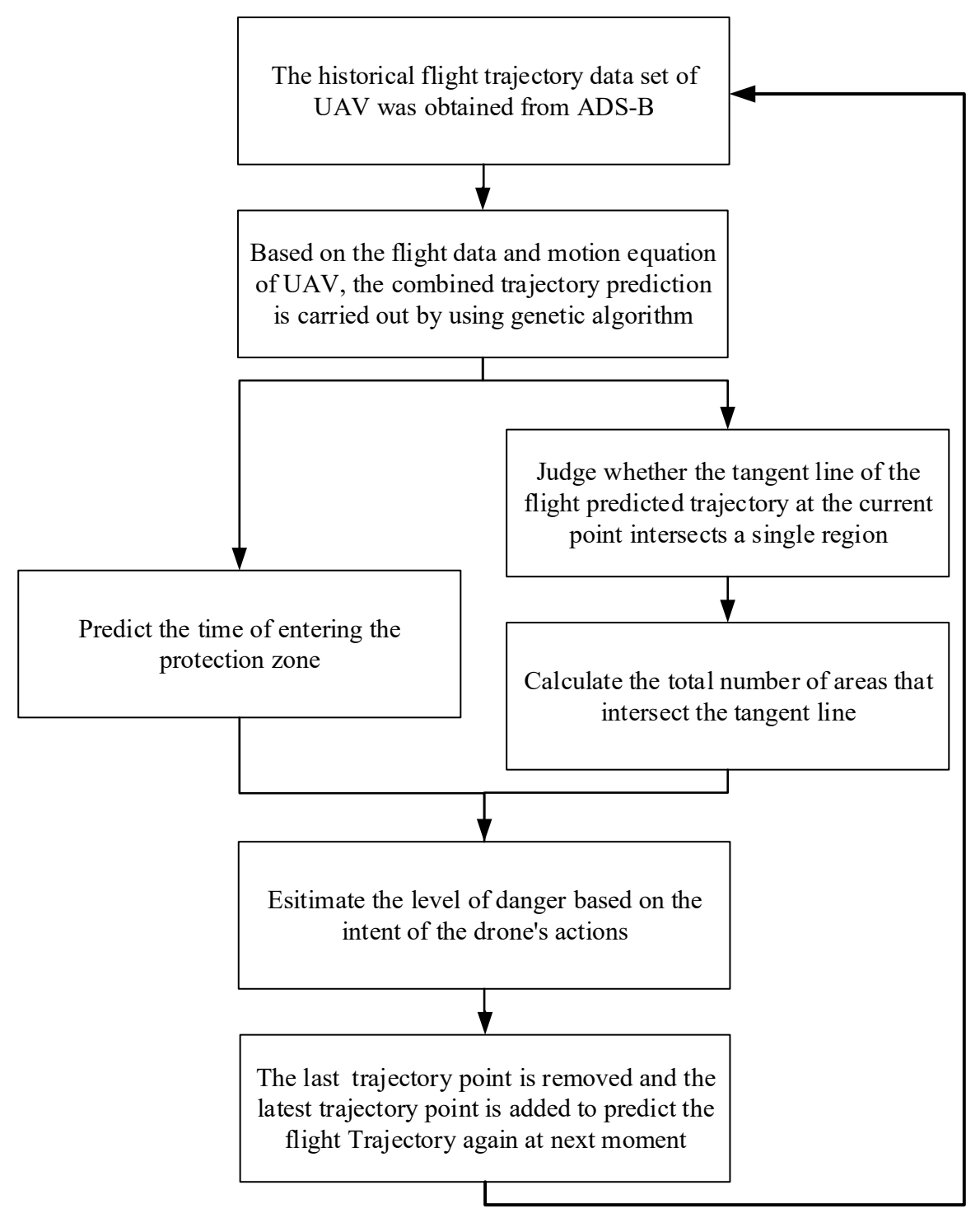

Figure 3. Flow chart of UAV behavior-intention estimation method based on 4-D flight-trajectory prediction.

\section{Simulation Verification}

In this study, a relatively wide area was selected for the experiment, and a specific area was selected to simulate the protection zone. The 3-D coordinates of the inflection point of the protection zone were accurately obtained through the corresponding measuring instruments. Then, the original data of UAV flight trajectory are collected by ADS-B, and they are substituted into the program code of the corresponding algorithm for simulation.

There are two criteria for the classification of dangerous UAV behavior: the number of areas where the tangent line of the predicted trajectory intersects with the collision area, alarm area, and alert area; the time when the UAV enter the protection area. Dangerous behaviors can be divided into eight grades $(1,2,3,4,5,6,7,8)$, from small to large, as shown in Table 1. 
Table 1. Discriminant criteria of UAV behavior intention.

\begin{tabular}{|c|c|c|c|c|}
\hline Time (s) Number of Interse & 0 & 1 & 2 & 3 \\
\hline$t \leq 240$ & 6 & 6 & 8 & 8 \\
\hline $240 \leq t<300$ & 5 & 5 & 7 & 7 \\
\hline $300 \leq t<360$ & 2 & 2 & 4 & 4 \\
\hline$t \geq 360$ & 1 & 1 & 3 & 3 \\
\hline
\end{tabular}

The classification standards of collision area, alarm area, and alert area are shown in Figure 4. The collision area is the protection zone, the alarm area is within $500 \mathrm{~m}$ of each side of the collision area, and the alert area is within $500 \mathrm{~m}$ of each side of the alarm area.

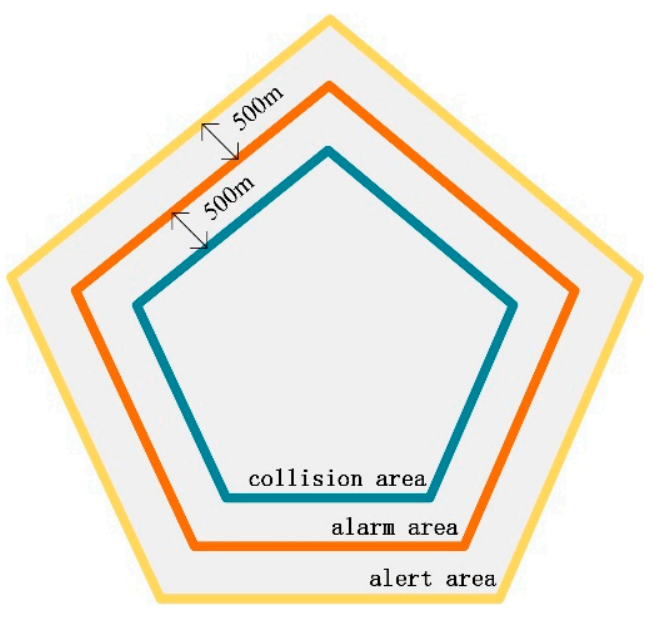

Figure 4. Division standards of collision area, alarm area, and alert area.

\subsection{Trajectory-Prediction Simulation}

The geographical scope of the simulation environment is $113^{\circ} 46^{\prime}$ to $114^{\circ} 37^{\prime} \mathrm{E}, 22^{\circ} 27^{\prime}$ to $22^{\circ} 52^{\prime} \mathrm{N}$. The original data of the UAV trajectory can be obtained through ADS-B, and the true flight trajectory can be drawn, as shown in the Figure 5.

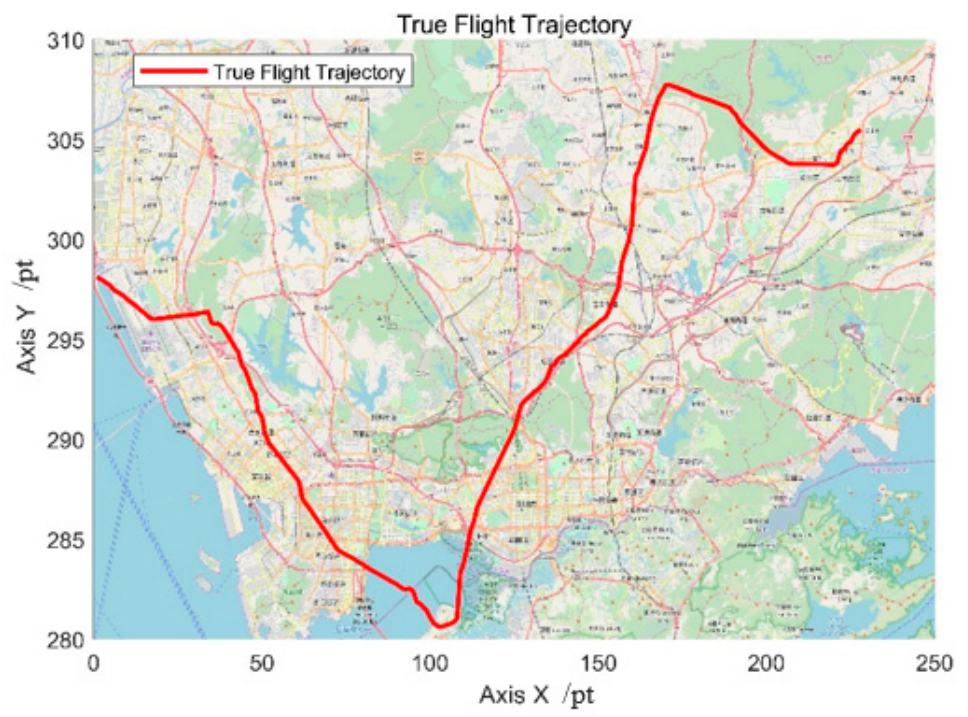

Figure 5. UAV trajectory based on raw data.

According to the combined prediction results of SVR and motion equation, the predicted flight trajectory is plotted, as shown in the Figure 6. 


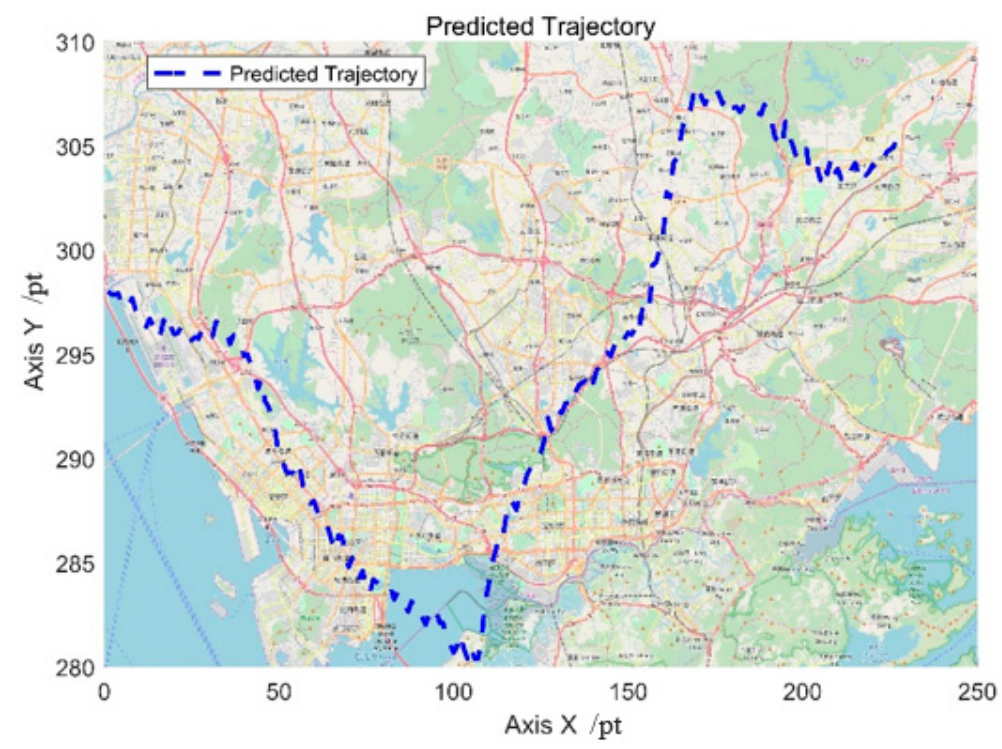

Figure 6. Combined trajectory prediction based on genetic algorithm.

In order to verify the rationality of the simulation process, a comparative analysis should be made between the combined predicted trajectory and the true flight trajectory. The comparison result is shown in the Figure 7.

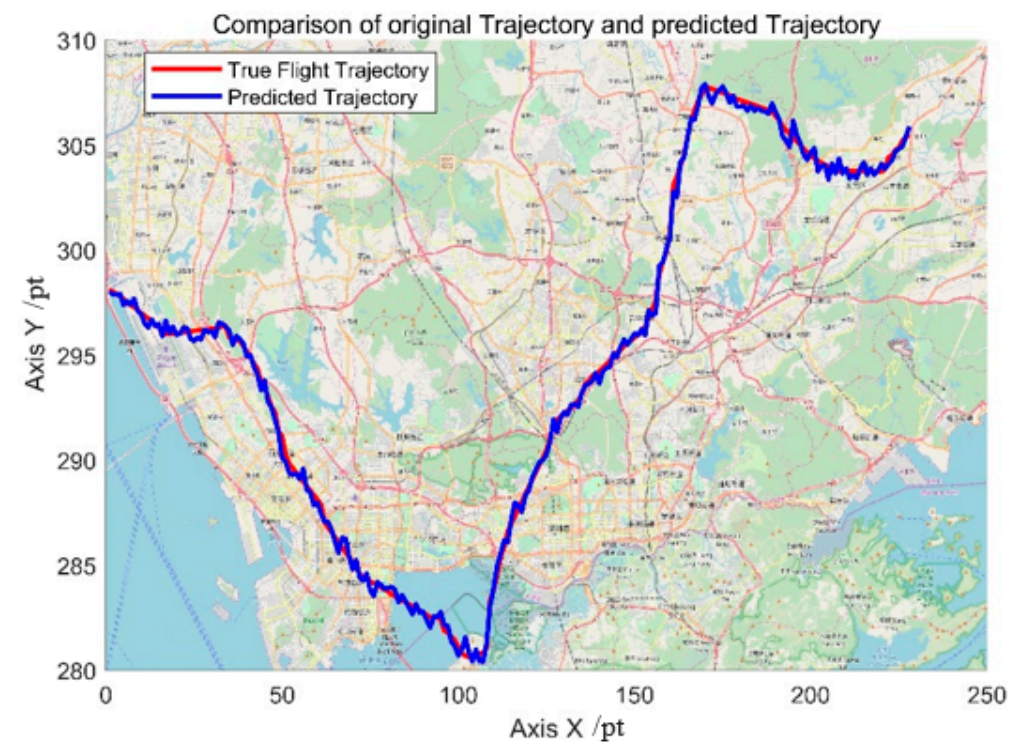

Figure 7. Comparison of original trajectory and predicted trajectory.

The rationality of the prediction algorithm and the correctness of the simulation process can be verified by deviation analysis of the predicted trajectory value and the real trajectory value. The deviation analysis of trajectory prediction is shown in the Figure 8. 


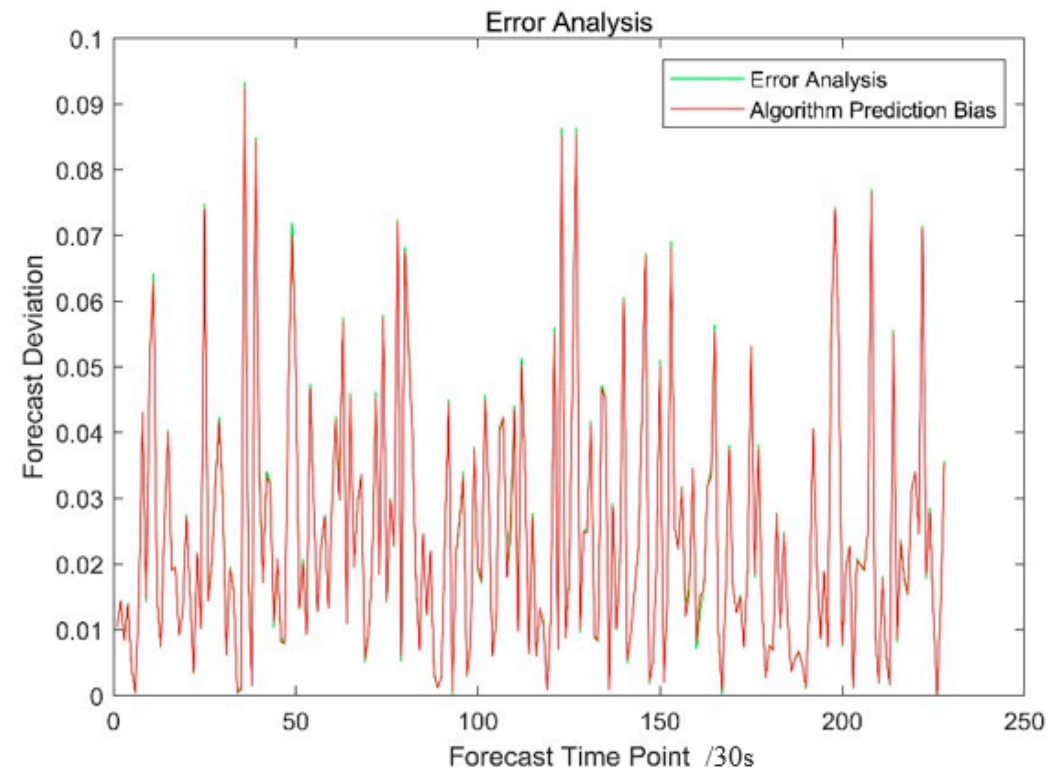

Figure 8. Analysis of UAV position deviation.

As can be seen from the figure, the maximum deviation is 0.1025 , the mean deviation is 0.0247 , the mathematical expectation is 0.0247 , and the mean square deviation is $3.6483 \times 10^{-4}$.

\subsection{Behavior-Intention Estimation Simulation}

In order to estimate the behavior intention of the UAV, the tangent line of the coordinate point of the flight trajectory-fitting curve at the current moment is obtained. The danger level is judged according to the number of intersections between the tangent line and the collision area, alarm area, and alert area, and the estimated time of entering the protection zone. Simulation images in danger levels 6 and 8 are shown in the Figures 9 and 10.

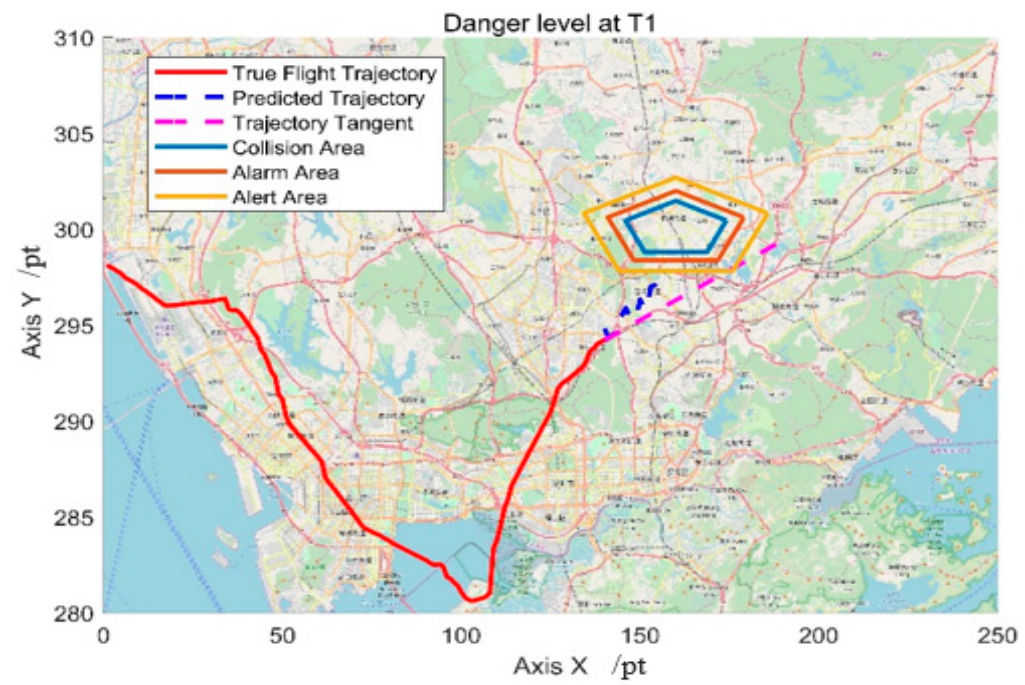

Figure 9. The danger level of UAV is 6.

As can be seen from the simulation results, when the flight trajectory is like the red curve, the intersection points of the tangent line of the current trajectory point and the collision area, alarm area, and alert area at $t_{1}, t_{2}$ are 0,3 , respectively, and the time of entering the protection zone is less than $240 \mathrm{~s}$. It can be seen that in these situations, the danger level is gradually increasing, and corresponding maneuvering behaviors should be taken to relieve the conflict. 


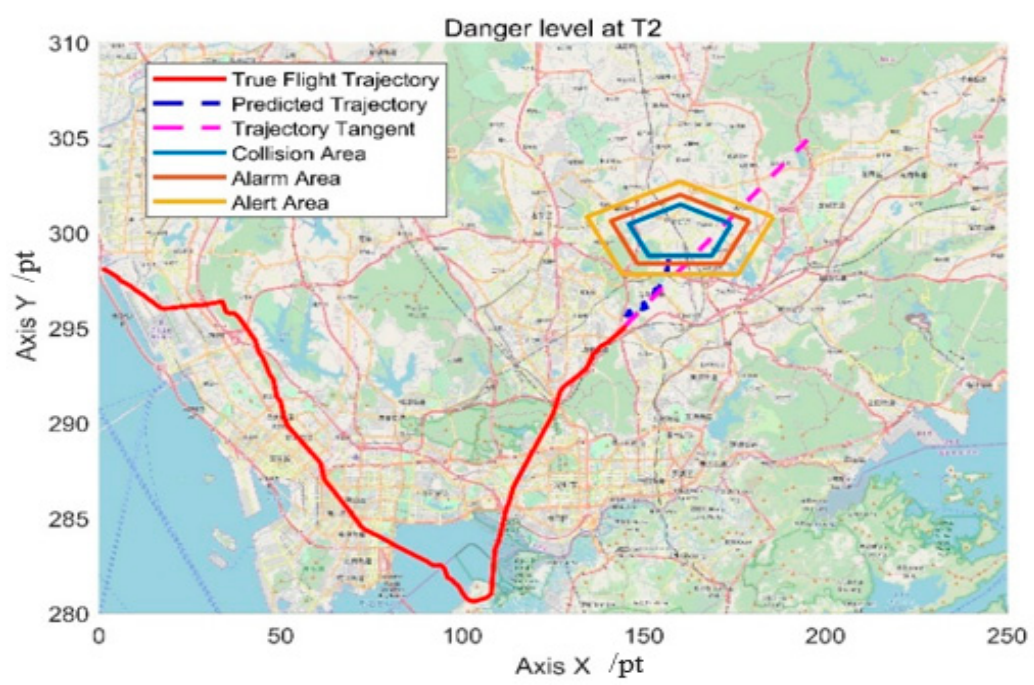

Figure 10. The danger level of $U A V$ is 8.

\section{Conclusions}

In this paper, the deficiencies of the existing 4-D trajectory-prediction models of UAV are analyzed, and a 4-D trajectory combined-prediction model based on genetic algorithm is proposed, based on which the dangerous behavior intention of UAV is estimated. The model can accurately predict the trajectory of UAV and the time when it enters the protection zone in real time and provide reference for the subsequent maneuvering behavior. However, this model has some shortcomings: the prediction model only analyzes the historical trajectory data and the kinematics of the UAV and does not consider the dynamic characteristics of the UAV; only the number of intersecting areas and the time of entering the protection zone are considered in the danger-behavior estimation, which has some limitations.

Author Contributions: Conceptualization, H.Z.; methodology, H.Z.; software, Y.Y.; data curation, S.L.; writing—original draft preparation, Y.H.; writing—review and editing, H.L. All authors have read and agreed to the published version of the manuscript.

Funding: This research was funded by National Natural Science Foundation of China (71971114) and the State Key Laboratory of Air Traffic Management System and Technology (SKLATM201801).

Institutional Review Board Statement: Not applicable.

Informed Consent Statement: Not applicable.

Data Availability Statement: Not applicable.

Acknowledgments: The authors acknowledge, with thanks, the support for this work of the College of Civil Aviation at Nanjing University of Aeronautics and Astronautics.

Conflicts of Interest: The authors declare no conflict of interest.

\section{References}

1. Prevot, T.; Rios, J.; Kopardekar, P.E.; Robinson, J.; Johnson, M.; Jung, J. UAS Traffic Management (UTM) Concept of Operations to Safely Enable Low Altitude Flight Operations. In Proceedings of the 16th AIAA Aviation Technology, Integration, and Operations Conference, Washington, DC, USA, 10 June 2016.

2. Rennong, Y.; Longfei, Y.; Min, S.; Xiaojian, C.; Xin, W. UVA Trajectory Prediction Model and Simulation Based on Bi-LSTM. Adv. Aeronaut. Sci. Eng. 2020, 11, 77-84.

3. Benavides, J.V.; Kaneshige, J.; Sharma, S.; Panda, R.; Steglinski, M. Implementation of a trajectory prediction function for trajectory based operations. In Proceedings of the AIAA Atmospheric Flight Mechanics Conference, Atalnta, GA, USA, 13 June 2014.

4. Qinggang, W. Research on Trajectory Prediction Technology of Mission-oriented Cooperating Unmanned Aircraft. Ph.D. Thesis, Nanjing University of Aeronautics and Astronautics, Nanjing, China, 2019. 
5. Shaojun, L.; Xing, Z.; Lipeng, X.; Dongsheng, L. Muti-Condition state prediction of longitudinal control loop of fixed-wing UAV. J. Ordnance Equip. Eng. 2020, 41, 203-209.

6. Teng, L.; Xinmin, T. UAV dynamic geofence planning method based on flexible four-dimensional trajectory prediction. Aeronaut. Comp. Technol. 2019, 49, 79-84.

7. Wang, Y.; Wang, J.; Xue, Y. UAV attitude estimation algorithm and its FPGA implementation based on pipeline gaussian particle filter. Electron. Opt. Control. 2019, 26, 66-70.

8. Tastambekov, K.; Puechmorel, S.; Delahaye, D.; Rabut, C. Aircraft trajectory forecasting using local functional regression in sobolev space. Transp. Res. Part C Emerg. Technol. 2014, 39, 1-22. [CrossRef]

9. Hong, S.; Lee, K. Trajectory prediction for vectored areanavigation arrivals. J. Aerosp. Inf. Syst. 2015, 12, 490-502.

10. Jiao, L.; Guoyou, S.; Xueqian, Y.; Kaige, Z. Ship trajectory prediction model based on DE-SVM. J. Shanghai Marit. Univ. 2020, 41, 34-39.

11. Shifei, D.; Bingjuan, Q.; Hongyan, T. An overview on theory and algorithm of support vector machines. J. Univ. Electron. Sci. Technol. China 2011, 40, 2-10.

12. Ning, W.; Min, X.; Jialiang, D.; Mingbo, L.; Jialong, L.; Yi, W.; Sijie, L. Mid-long term temperature-lowering load forecasting based on combination of support vector machine and multiple regression. Power Syst. Prot. Control 2016, 44, 92-97.

13. Zhixuan, F. An analysis of track prediction based on kalman filter. Sci. Tech. Inf. Gansu 2019, 48, 33-36.

14. Yan, L.; Zenggang, X.; Ailing, C. Tourism passenger flow prediction method based on multi-scale combination. Stat. Decis. 2020, 36, 177-180.

15. Yanshuo, T. Research and Application of Genetic Algorithm. Master's Thesis, University of Electronic Science and Technology of China, Chengdu, China, 2004.

16. Kezong, T.; Tingkai, S.; Jingyu, Y. An improved geneticalgorithm based on a novel selection strategy for nonlinear programming problems. Comp. Chem. Eng. 2011, 35, 615-621. 